\title{
Active control of periodic fan noise in laptops: spectral width requirements in a delayed buffer implementation
}

\author{
H. A. Cordourier-Maruri* ${ }^{1}$, F. Orduña-Bustamante ${ }^{2}$ \\ ${ }^{1,2}$ Laboratorio de Acústica y Vibraciones, CCADET \\ Universidad Nacional Autónoma de México. \\ *cordourier@hotmail.com
}

\begin{abstract}
An active control system intended for the reduction of strictly periodic noise components in computer cooling fans is described, which is based on high-performance digital sound device architectures found in some personal computers. The system overcomes causality and synchronization constrains imposed by delayed buffering, as usually found in computer audio processing. Performance of the system is demonstrated and evaluated through measurements in a physical implementation of active noise control of synthetic tones combined with laptop fan noise, carried out under anechoic and slightly reverberant conditions. Tests on other types of tonal noise sources, like an electrical transformer, were also carried out. However, its wider applicability to the cancellation of tonal noise has been proved compromised by weak periodicity issues found and reported in this work. Also, a study of noise spectral width requirements for successful operation is presented.
\end{abstract}

Keywords: Computer noise, fan noise, tonal noise, periodic noise, computer cooling fan, active noise control.

\section{RESUMEN}

Se describe un sistema de control activo para la reducción de las componentes de ruido periódico en ventiladores pequeños de computadora. El sistema se basa en arquitecturas de sonido digital de alto desempeño, presentes en algunas computadoras personales. El sistema es capaz de superar las limitaciones de causalidad y sincronización provocadas por retardos en la operación basada en bloques de datos (audio buffers), que es usual en el procesamiento digital de audio en computadora. El desempeño del sistema se demuestra a través de mediciones en una implementación física de control activo de ruido de tonos sintéticos combinados con el ruido de ventilador de una computadora portátil. Estas mediciones fueron llevadas a cabo bajo condiciones acústicas anecoicas y ligeramente reverberantes. También se llevaron a cabo pruebas con otras fuentes típicas de ruido tonal, como un transformador eléctrico. Sin embargo, se ha probado que la capacidad de aplicación para cancelar ruido tonal está limitada por pequeñas variaciones en la periodicidad del ruido, determinadas y reportadas en este trabajo. Se presenta también un estudio experimental de los requerimientos en la anchura espectral del ruido para una operación exitosa.

Palabras clave: Ruido de computadora, ruido de ventilador, ruido tonal, ruido periódico, ventilador de computadora, control activo de ruido.

\section{Introduction}

The main source of continuous noise generated by personal computers is normally the external cooling fan. However, ventilation has become progressively indispensable in PCs, due to the ever increasing heat dissipation requirements of smaller (denser) and faster integrated processing circuits [1]. It is widely understood that Active Noise Control (ANC) is capable of notably reducing global noise levels when acting from locations close to the source, without interfering with the source mechanism; both of which are advantages not found with passive control strategies $[2,3,4]$. Most research and development in this area has focused on reduction of fan noise tonal components, since they represent the main source of acoustic annoyance for users $[1,5]$.

Performance of ANC strategies for fan noise reduction varies widely with application. Reductions of sound power with attenuation of 5 to $7 \mathrm{~dB}$ were obtained in an axial fan by Gérard et al. $[6,7]$ with one speaker located in the middle of the fan. Wu and Bay [8] achieved $13 \mathrm{~dB}$ of Sound 
Pressure Level (SPL) attenuation of the main tonal component (BPF, Blade Passing Frequency). A larger level of reduction is found in a hybrid system (passive and active control combined) that was tested by Wong et al. [9], which managed to obtain SPL reductions up to $23 \mathrm{~dB}$ of the BPF in a room ventilation fan. These implementations were based on dedicated DSP hardware architectures.

\section{Active noise control through computer audio hardware}

In this work, we consider a single-channel active noise control system with one reference signal, one control signal, and one error signal $[10,11$, 12], as shown in Figures 1 and 2. Symbols for signals and transfer functions are defined as follows:

- $s[n]$ : reference signal picked up by microphone $A$, close to the noise source.
- $r[n]:$ modified reference signal, without feedback from the control signal

- $q[n]$ : control signal fed to the control sound source (loudspeaker).

- $q_{s}[n]$ : control signal transferred to the reference microphone $A$, used for feedback cancellation.

- $p[n]$ : control signal transferred to the error microphone $B$.

- $u[n]$ : noise signal to be canceled at the error microphone $B$.

- $e[n]$ : error signal to be minimized, picked up by microphone $B$, at the ear, as the superposition of the transferred control signal and the noise signal.

- $H(z)$ : transfer function of control filter.

- $F(z)$ : transfer function from control signal to reference microphone $A$ (feedback path).

- $G(z)$ : transfer function from control signal to error microphone B.
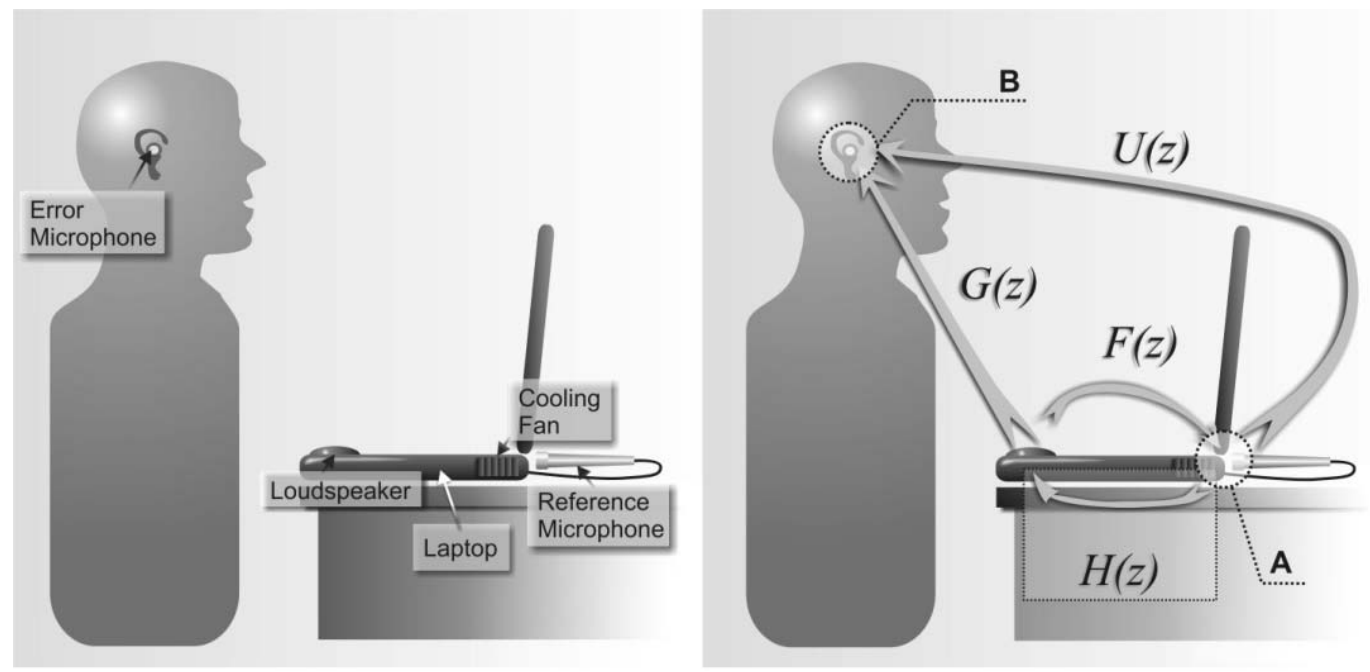

Figure 1. Active noise control in a laptop computer. Illustration showing system elements (left), and transfer functions (right). Note labels of reference microphone A, and error microphone B. 


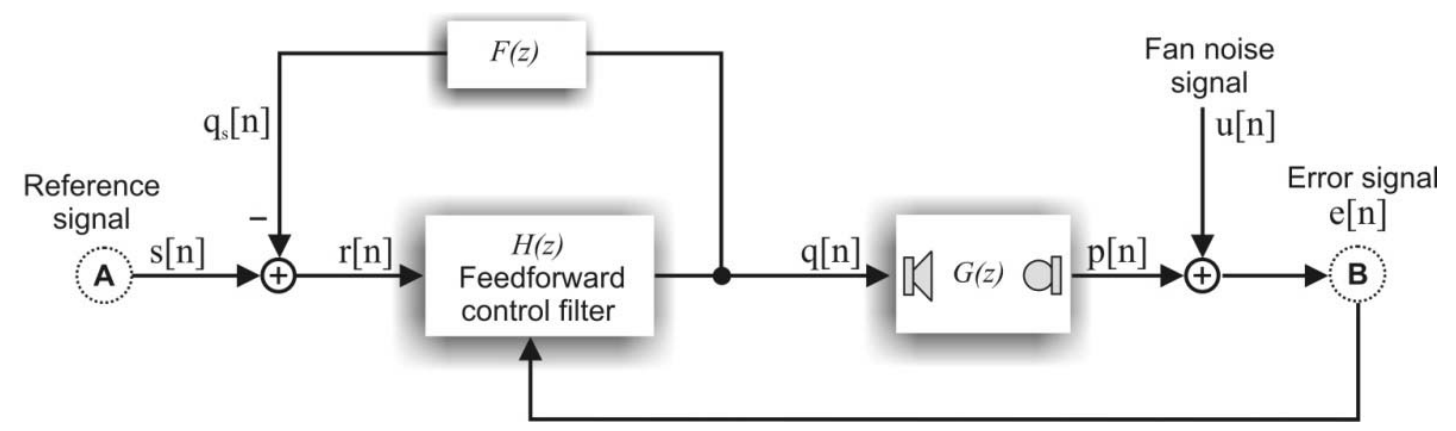

Figure 2. Block diagram of feedforward controller implemented on a standard computer audio system, implying delayed audio buffering (not shown) of physical inputs and outputs.

The coefficients of the non-recursive FIR control filter $H(z)$ are updated using the filtered-x block LMS algorithm with feedback cancellation, implemented in the frequency domain $[13,14]$. However, the algorithm must be modified in order to perform properly on the proposed implementation in standard computer audio hardware.

Computer audio processing is invariably implemented by buffering audio blocks of $N$ samples, with typical block sizes $N$ ranging from 64 to 4096 or more samples. Simultaneous recording and playback of audio buffers, implies that the application software must deal with a delay of at least $N$ samples between the corresponding physical input/output signals. In some cases, the software driver can induce a block delay in multiples of the block size: $2 N, 3 N$, etc., instead of $N$. An additional delay is usually involved, having to do with signal conditioning (AD/DA conversion, hardware $[11,15]$. Under the strong assumption that the tonal disturbance is strictly periodic, with period $T$ samples (possibly fractional), then delayed audio buffering can, in principle, be overcome [16].
The delays between the actual physical signals and the digital signals that are being simultaneously processed by the CPU, which affect the proposed noise control system, can be classified as follows:

$N_{i}=$ Delay samples of digital input signal, relative to physical input.

$N_{o}=$ Delay samples of physical output signal, relative to digital output.

Analysis of these delays is illustrated in Figure 3(a). An equivalent, simplified scheme of the system, with delays relocated in series with $H(z)$ and $F(z)$ transfer functions, is illustrated in Figure $3(b)$. Since an internal delay $D$, due to signal conditioning, can be assumed identical at the input and output stages (mainly related to antialias and reconstruction low-pass filters of the same order), $\mathrm{Ni}$ and $\mathrm{No}$ can be expressed as follow:

$$
\begin{aligned}
& N_{i}=D \\
& N_{o}=N+D
\end{aligned}
$$




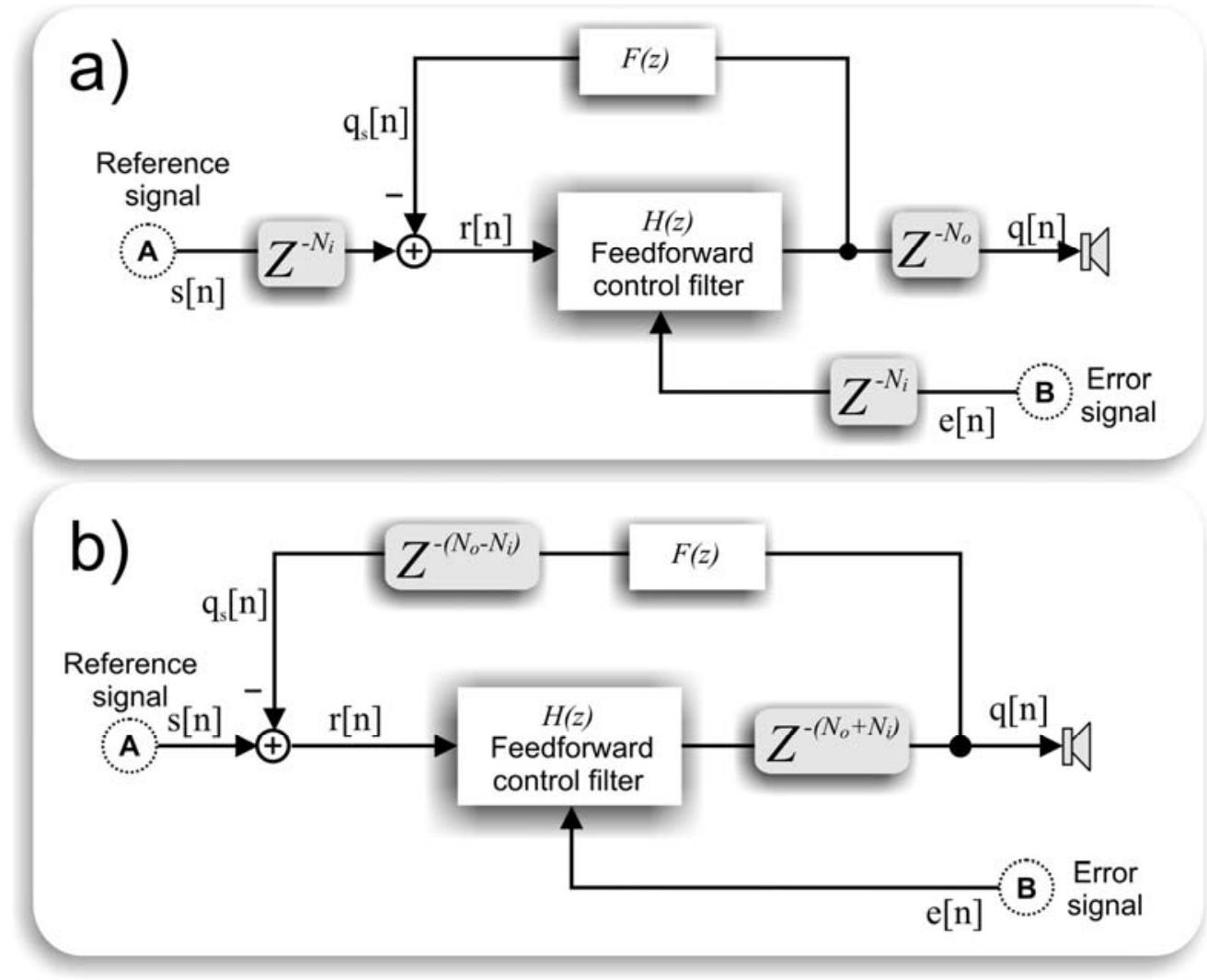

Figure 3. Block diagrams of controller with delay blocks associated with computer internal buffering and signal conditioning (grey). Diagram (a) shows delay blocks at physical input and output stages. Diagram (b) is an equivalent diagram, with delays relocated in series with $F(z)$ and $H(z)$ transfer functions.

Where $\mathrm{N}$ is the block delay, and $\mathrm{D}$ is the signal conditioning delay. Substituting these values in the simplified block scheme shown at Figure 3(b), results in $\mathrm{F}(\mathrm{z})$ being delayed $\mathrm{N}$ samples, and $\mathrm{H}(\mathrm{z})$ being delayed $\mathrm{N}+2 \mathrm{D}$ samples. Implications of delayed audio processing to the implementation of active control of tonal noise are illustrated in Figure 4.

Control signals calculated from delayed reference signals must be synchronized to the tonal noise period T, as illustrated in Figure 5. Also, feedback cancellation of the control signal at the reference microphone needs to be properly synchronized to the period T, as illustrated in Figure 6.
Modifications required to the filtered-x block LMS algorithm with feedback cancellation, can be summarized as follows:

- The control filter $H(z)$ must be delayed by $N+$ 2D mod $T$ samples.

- The feedback filter $F(z)$ must be delayed by $N$ $\bmod T$ samples, for a total delay of $2 N+2 D \bmod T$ samples, as it operates on the output of the control filter, already delayed $N+2 D \bmod T$ samples.

Two final implications, are that the tonal noise period $T$ must be known, or estimated during processing $[17,18]$, and the audio delays $N$ and $D$ must be previously identified from measurements in the audio hardware. 

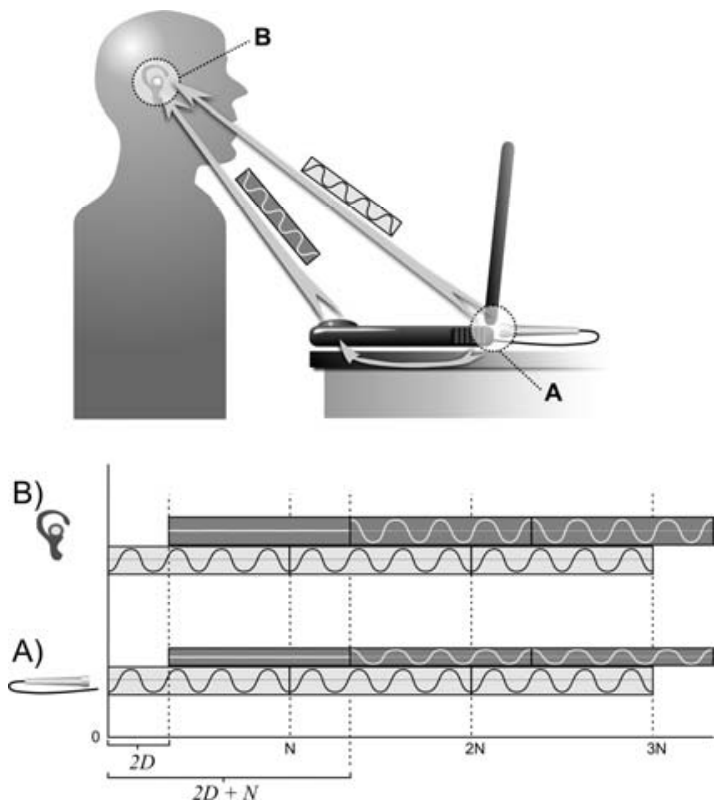

Figure 4. Delayed audio buffering of control signal (dark gray), and noise signal (light gray), at reference $A$ and error $B$ microphones (acoustic delays not shown for simplicity). Total audio delay $N_{i}+N_{o}=N+2 D$ includes a block delay of $N$ samples, and an internal audio system delay of $2 D$ samples (signal conditioning at input and output).

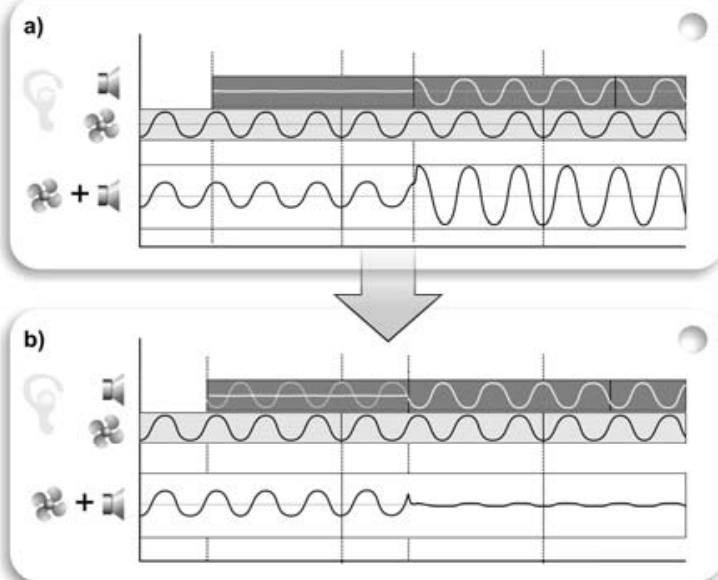

Figure 5. Superposition of acoustic signals at the ear (error microphone), in two cases: a) audio delay producing possibly incorrect cancellation, b) tone-synchronized audio compensation $(H(z)$ is delayed $N+2 D \bmod T$ samples) ensuring correct cancellation).
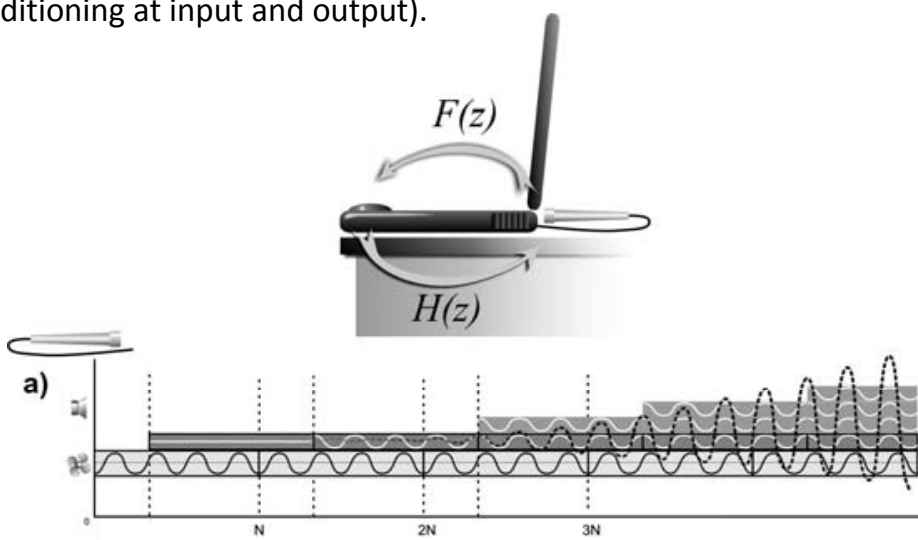

b)

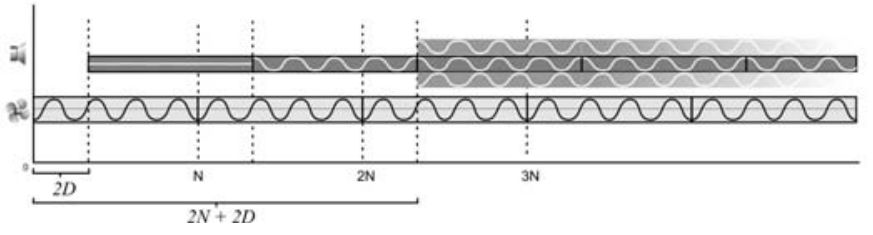

Figure 6. Feedback cancellation of the control signal at the reference microphone, in two cases: a) audio delay leading to unstable feedback, b) tone-synchronized audio compensation $(F(z)$ is delayed $2 N+2 D$ mod $T$ samples) ensuring stable feedback cancellation. 


\section{Experimental setup}

An active noise control system based on standard audio hardware, as described in the previous section, was implemented using the following equipment:

- Laptop computer 1 , based on a Pentium 4 at 2 $\mathrm{GHz}, 512 \mathrm{MB}$ RAM, executing a CPU intensive numeric routine to keep it hot, working at full capacity, and with the cooling fan running at, or near, maximum speed. This laptop was used only as a source of fan noise for convenience, although it could have been running its own active noise control algorithm just as well.

- Laptop computer 2, based on a Pentium 4 at 3 $\mathrm{GHz}, 512 \mathrm{MB}$ RAM, used for audio processing (audio input/output, automatic identification of main tonal noise component, and active noise control algorithm). This was implemented in Matlab 6.5, using Matlab's Data Acquisition Toolbox for real time audio $1 / O$ through an external audio interface (with implicit delayed audio buffering).

- External audio interface, MOTU 896-HD, with Firewire (IEEE-1394) connection to audio processing laptop 2.
- Two audio measurement condenser microphones, Behringer ECM-8000; one was used as reference microphone $A$, the other as error microphone $\mathrm{B}$.

- Amplified external computer loudspeaker (single audio channel), based on a small two-inch cone driver, this was placed at about $30 \mathrm{~cm}$ from laptop's 1 cooling fan (and from reference microphone A), and at about $40 \mathrm{~cm}$ from error microphone $B$.

- Cellular telephone placed very close to laptop's 1 cooling fan, used to remotely generate a WAV recording of a long stable $700 \mathrm{~Hz}$ harmonic tone, included as a synthetic tonal noise component in some of the active noise control tests.

- Electrical 2000 VA three-phase transformer Sola Basic $\mathrm{CVH}$, as an alternative tonal noise source [19].

- Electrical 1200 VA voltage regulator Sola Basic, also as an alternative tonal noise source.

Figure 7 shows an illustration of the experimental setup used for the active noise control tests. These were carried out in a small classroom (volume $V \approx$ $70 \mathrm{~m}^{3}$, reverberation time $T_{60} \approx 0.7 \mathrm{~s}$ at $1 \mathrm{kHz}$ ), and also in an anechoic chamber (free volume $\mathrm{V} \approx 50$ $\mathrm{m}^{3}$, lower anechoic frequency $\mathrm{f}_{\mathrm{c}} \approx 70 \mathrm{~Hz}$ ).

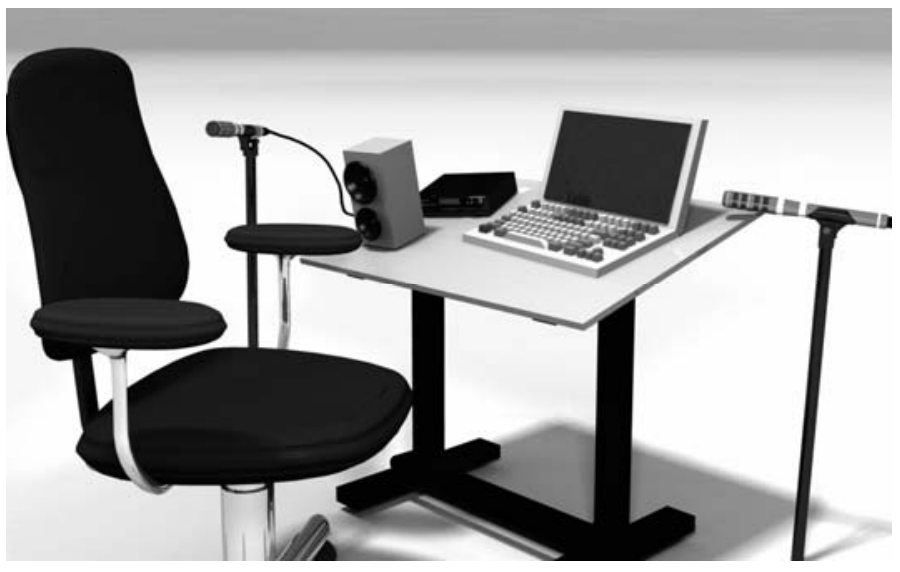

Figure 7. Illustration of an active noise control system in practice, similar to the experimental setup used in this work (except that error microphone A, shown here at the right-hand side of the laptop, was actually placed at the equivalent position on the left-hand side, closer to the fan also at left, and to the control source). 


\section{Results}

Figure 8 (a) shows noise spectra from Laptop computer 1 , at the error microphone $B$, with and without active control, and noise reduction level, measured in a small classroom. Laptop noise containing tonal and broadband noise was complemented by a $700 \mathrm{~Hz}$ synthetic tone generated by a cellular phone hanging very close to the laptop's cooling fan. Active noise control based on computer audio hardware is able to successfully cancel the synthetic $700 \mathrm{~Hz}$ tone, with a reduction level of nearly $-30 \mathrm{~dB}$. Figure 8 (b) shows a similar situation but with laptop noise only. In this case, however, the active noise control system is unable to cancel the main tonal component at about $1270 \mathrm{~Hz}$.
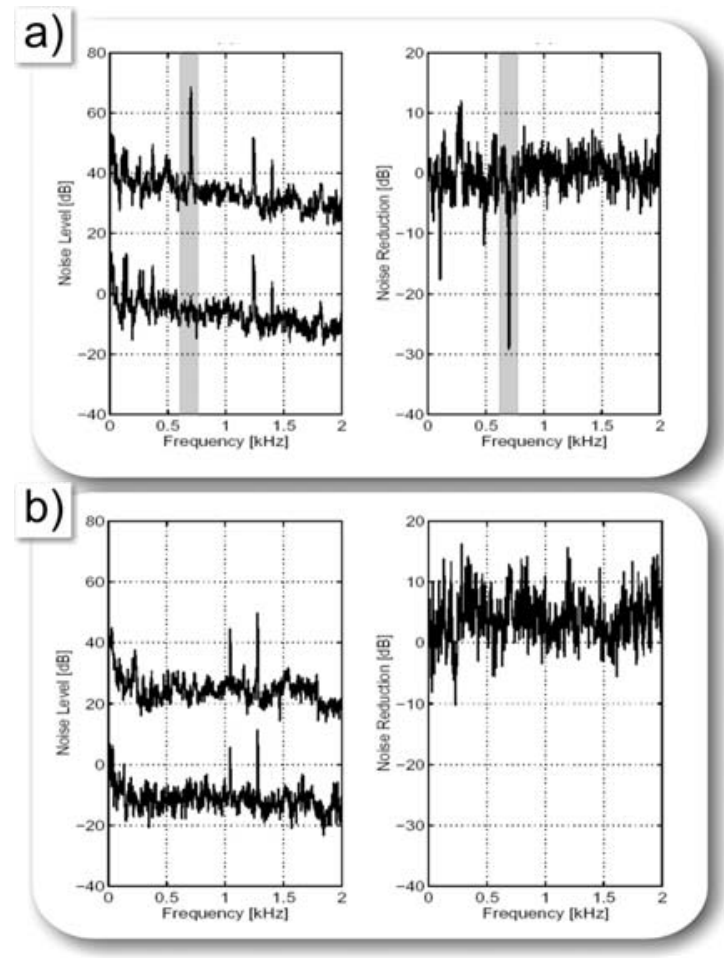

Figure 8. Active control of a $700 \mathrm{~Hz}$ synthetic tone and laptop noise (a), and laptop noise only (b) in a small classroom. Left side plots show noise levels without control (upper trace), with control (drawn $40 \mathrm{~dB}$ down for clarity). Right side plots show noise reduction level.
Figure 9 shows noise spectra from the 2000 VA electrical transformer, at the error microphone $B$, with and without active control, and noise reduction level, measured in an anechoic chamber. The active noise control system is only able to affect the $120 \mathrm{~Hz}$ tonal component, with a reduction level of nearly $-12 \mathrm{~dB}$.

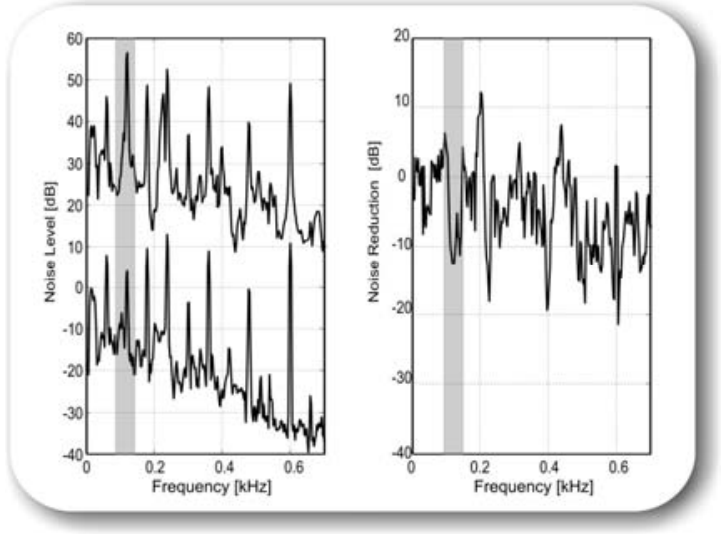

Figure 9. Active control of 2000 VA transformer noise, measured in an anechoic chamber. Left side plot show noise levels without control (upper trace), and with control (drawn $40 \mathrm{~dB}$ down for clarity). Right side plot shows noise reduction level. Note specific noise reduction of $120 \mathrm{~Hz}$ component (highlighted).

In the same way, figure 10 shows noise spectra from the 1200 VA voltage regulator, at the error microphone $B$, with and without active control, and noise reduction level, measured in an anechoic chamber. Here, the active noise control system only affects the $480 \mathrm{~Hz}$ tonal component, with a reduction level of nearly $-13 \mathrm{~dB}$.

Figure 11 shows a high definition spectral analysis of laptop fan noise measured at error microphone $B$ in an anechoic chamber. Zooming in around the $844 \mathrm{~Hz}$ component reveals a lack of strictly tonal character, a peak with a spectral width of about 6 $\mathrm{Hz}$, possibly produced by multiple components of similar frequency, and/or by slowly modulated. 

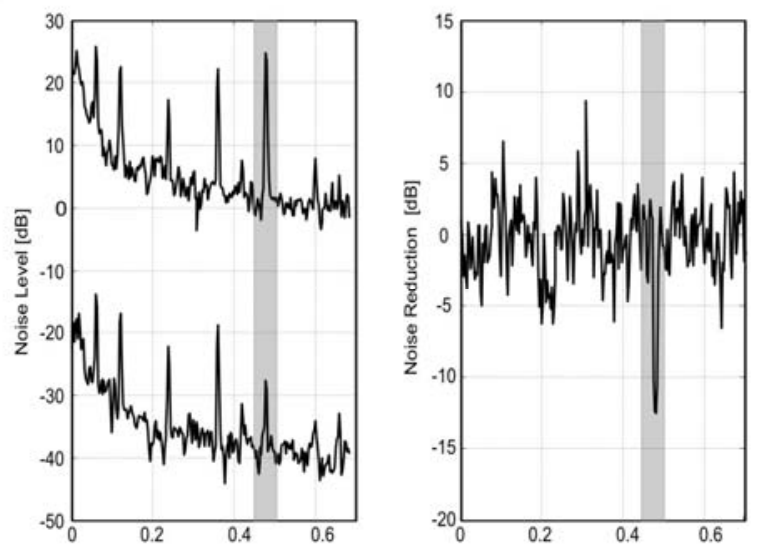

Figure 10. Active control of 1200 VA regulator noise, measured in an anechoic chamber. Left side plot shows noise levels without control (upper trace), and with control (drawn $40 \mathrm{~dB}$ down for clarity). Right side plot shows noise reduction level. Note specific noise reduction of $480 \mathrm{~Hz}$ component (highlighted).

a)

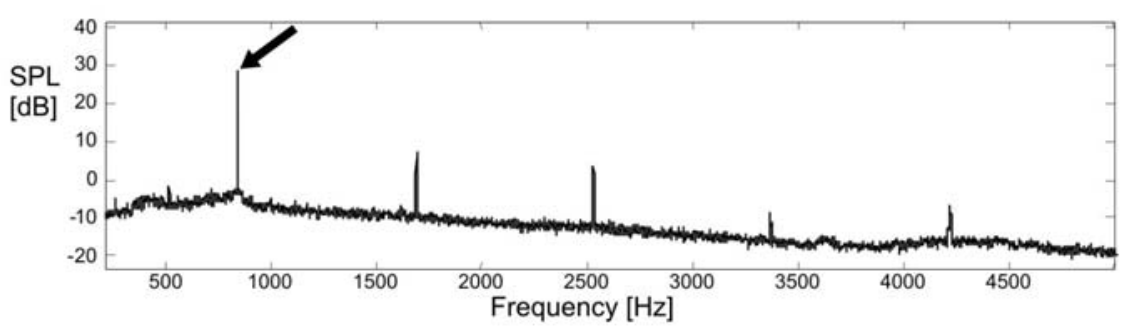

b)

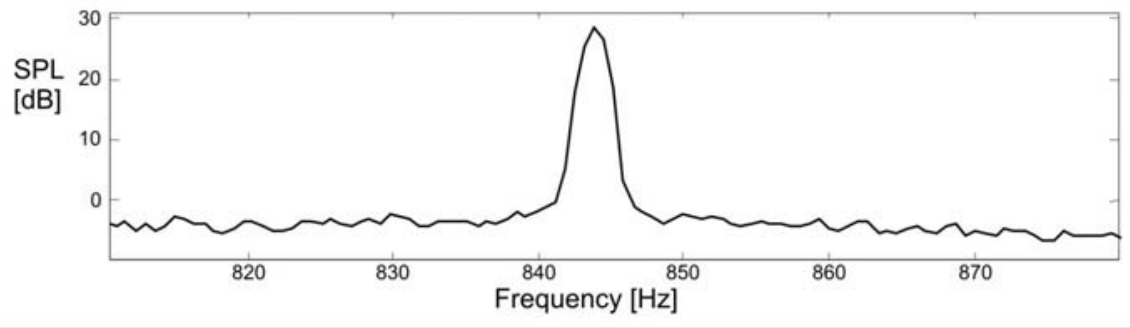

Figure 11. Spectral analysis of sound pressure level generated by laptop fan noise at error microphone B in an anechoic chamber: (a) 0 to $5000 \mathrm{~Hz}$ broadband analysis (844 Hz component indicated by small arrow), (b) $70 \mathrm{~Hz}$ zoom analysis around $844 \mathrm{~Hz}$ component "tone". Note spectral width of about $6 \mathrm{~Hz}$ over noise floor. 
components [8]. This seemingly negligible deviation from strict periodicity proves to be crucial. Spectral width of tonal noise components $\Delta f$ is calculated by first obtaining the frequency centroid:

$$
f_{c}=\frac{\sum_{n=N_{1}}^{N_{2}} f_{n} \cdot S\left(f_{n}\right)}{\sum_{n=N_{1}}^{N_{2}} S\left(f_{n}\right)}
$$

And then, calculating the standard deviation around it:

$$
\Delta f=\sqrt{\frac{\sum_{n=N_{1}}^{N_{2}}\left[f_{n}-f_{c}\right]^{2} \cdot S\left(f_{n}\right)}{\sum_{n=N_{1}}^{N_{2}} S\left(f_{n}\right)}}
$$

Where $f_{n}$ are frequency values, and $S\left(f_{n}\right)$ is the power spectral amplitude of the noise at frequency $f_{n}$. In order to analyze only one tonal component, a small range of elements from $N_{1}$ to $N_{2}$ around the central tonal frequency is considered in the sums.

Standard deviation measurements were taken of main periodic noise components of all different noise sources considered. Results of these measurements are shown in Figure 12. The lowest standard deviation components $(120 \mathrm{~Hz}$ for the 2000 VA transformer, $480 \mathrm{~Hz}$ for the $1200 \mathrm{VA}$ regulator and $700 \mathrm{~Hz}$ synthetic tone, all highlighted by arrows), are exactly the same components that where successfully reduced by the active noise control system in previous tests. Fan noise from laptop 1 was never affected, since its tonal spectral width was clearly out of range of effective operation of the system. This restriction compromises successful operation of the active noise control implemented through delayed audio buffering in its application to tonal fan noise cancellation in laptops. However, the technique presented can find applicability to other cases, in which the assumed strict periodicity can be met.

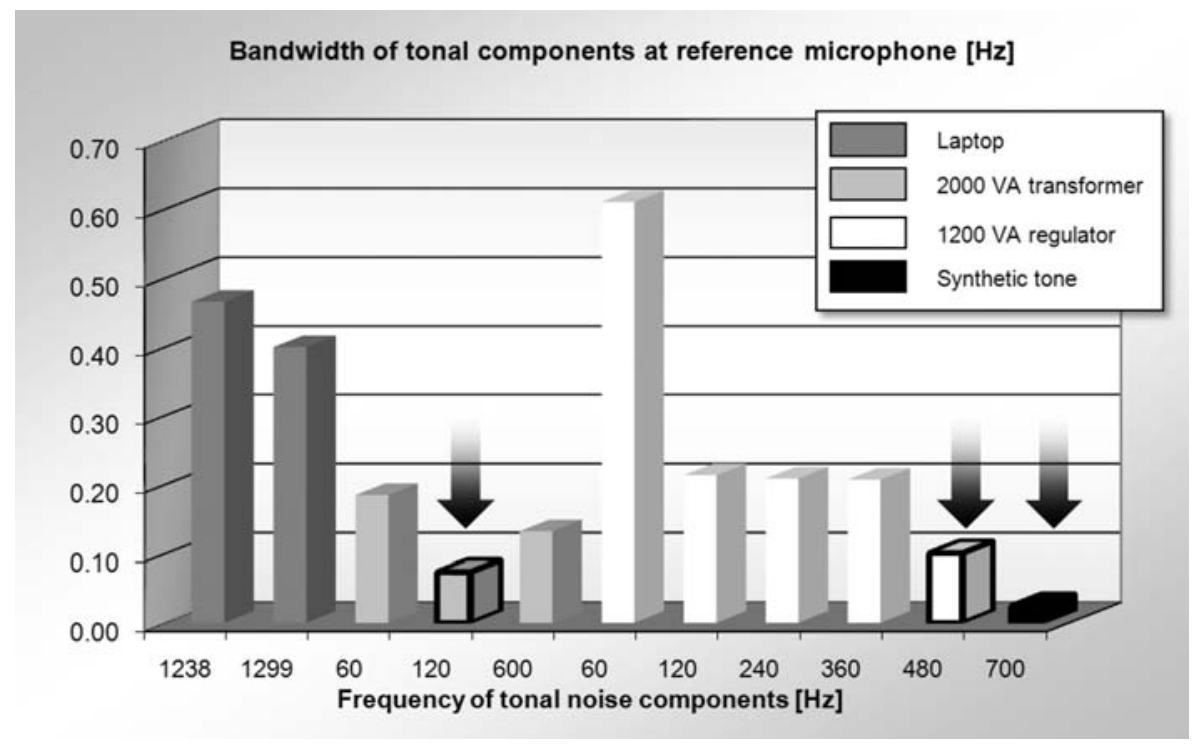

Figure 12. Spectral width measurement (standard deviation in hertz of spectral peak) of main tonal components of noise from different noise sources considered in previous active noise control tests. Smaller bandwidth components (highlighted with arrows) coincide with those successfully reduced by active noise control system. 


\section{Conclusion}

An active noise control system implemented in standard computer audio hardware has been described, that overcomes delayed audio buffering constrains by means of a modified filtered-x block LMS algorithm. The strategy has been proved suitable for the control of strictly periodic disturbances, but unsuccessful in situations where tonal noise components are weakly periodic. The case has been made of fan noise in a laptop, noise in an electrical transformer and noise of a voltage regulator, where it has been found that many "tonal" components exhibit non-negligible spectral widths, probably caused by multiple closely spaced, and/or slowly modulated, tonal components. The relationship between spectral width of the tonal components, and performance of the ANC algorithm was clearly disclosed as fundamental. This situation renders the proposed ANC strategy probably unsuitable for active cancellation of tonal noise from laptops, but remains a valid option for other applications in which tonal noise components exhibit tighter (more stable) periodicity bounds.

\section{References}

[1] Kinney, C. E., De Callafon, R. A., Dunens, E., Bargerhuff, R., Bash C. E., "Optimal periodic disturbance reduction for active noise cancellation", J. of Sound and Vibration, Vol. 305, No.1, August, 2007, pp. 22-33.

[2] Kuo, S. M., Morgan, D. R., "Active Noise Control: A Tutorial Review", Proceedings of the IEEE, Vol. 87, No. 6, June, 1999, pp. 943-973.

[3] Neise, W., Stahl, B., "Noise reduction in centrifugal fans: a literature survey", J. of Sound and Vibration, Vol. 45, No. 3, April 1976, pp. 375-403.

[4] Neise, W., "Review of noise reduction method for centrifugal fans", Journal of Engineering for Industry, Vol. 104, No. 2, May, 1982, pp. 151-161.
[5] Cattanei, A., Ghio, R., Bongiovi, A., "Reduction of the tonal noise annoyance of axial flow fans by means of optimal blade spacing", Applied Acoustics, Vol. 68, No. 11-12, November/December, 2007, pp. 1323-1345.

[6] Gérard, A., Berry, A., Masson, P., "Control of tonal noise from subsonic axial fan. Part 1: reconstruction of aeroacoustic sources from far-field sound pressure", J. of Sound and Vibration, Vol. 288, No. 4-5, December, 2005, pp 1049-1075.

[7] Gérard, A., Berry, A., Masson, P., "Control of tonal noise from subsonic axial fan. Part 2: Active control simulations and experiments in free field", J. Sound and Vibration, Vol. 288, No. 4-5, December, 2005, pp 10771104.

[8] Wu, J. D., Bay, M. R., "Application of feedforward adaptive active-noise control for reducing blade passing noise in centrifugal fans", J. of Sound and Vibration. Vol. 239, No. 5, February, 2001, pp. 1051-1062.

[9] Wong, Y. J., Paurobally, R., Pan, J., "Hybrid active and passive control of fan noise", Applied Acoustics. Vol. 64, No. 9, September, 2003, pp. 885-901.

[10] Widrow, B., Stearns, S., Adaptive Signal Processing (C-3), Prentice Hall, 1985.

[11] Nelson, P. A., Elliott, S. J., Active Control of Sound (C-6), Academic Press, 1993, pp 161-203.

[12] Clarkson, P. M., Optimal and Adaptive Signal Processing (C-4), CRC Press, 1993, pp. 205-274.

[13] Elliott S. J., Signal Processing for Active Control (C3), Academic Press, 2001, pp. 104-175.

[14] Hansen, C. H., Snyder, S. D. Active Control of Noise and Vibration. Cambridge University Press, 1997, pp 418-421.

[15] Hansen, C. H., Understanding Active Noise Cancellation. Spon Press, 2001, pp. 14-15. 
[16] Snyder, S. D., Active Noise Control Premier, Springer-Verlag, New York, Inc., 2000, pp. 63-65.

[17] Tavares Mancillas, A., Predicción lineal de término largo para la extracción de ruido tonal generado por ventiladores de computadora, MEng Thesis, Universidad Nacional Autónoma de México, 2006. Thesis supervisor: Orduña Bustamante, F.

[18] Orduña Bustamante, F., Tavares Mancillas, A., de la Guardia González, R. "Enhancement by linear prediction of acoustic reference signals for active noise control of computer cooling fans", Inter-Noise, 2006, Honolulu, Hawaii, USA, December.

[19] Qiu, X., Hansen, C. H., "An algorithm for active control of transformer noise with on-line cancellation path modeling based on the perturbation method", J. of Sound and Vibration, Vol. 240, No. 4, March, 2001, pp. 647-665.

\section{Acknowledgment}

Partial funding for this work has been granted by Intel Corporation, in support of research in the area of "Active noise control of small computer cooling fans close to the source" at Universidad Nacional Autónoma de México (UNAM). Participation of author Héctor Alfonso Cordourier Maruri has been additionally supported by a student grant from CEP-UNAM. This work is also a result of the partial support of Mexico Federal Government: Secretaría de Economía, CONACYT, and COECYTJAL. 


\section{Authors Biography}

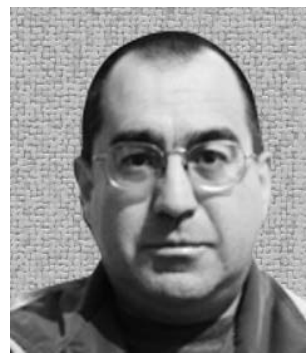

\section{Felipe ORDUÑA-BUSTAMANTE}

He is a Level A researcher at the Acoustics and Vibration Group, CCADETUNAM. He has a BSc degree in physics (UNAM, 1987), as well as MSc and PhD degrees in sound and vibration (University of Southampton, UK, 1990 and 1995). He works on active control of sound and vibration, digital signal processing, and musical acoustics, being author and coauthor of several journal and congress papers, technical reports, and international patents cited in the Science Citation Index. He is a fellow member of the Acoustical Society of America, and other academic societies. He is a professor and thesis supervisor of engineering and music at UNAM, actively participating in the creation of postgraduate study programs in scientific instrumentation (1998), and Music Technology (2004). He is an amateur musician, player of the classical guitar, baroque transverse flute, recorder, and keyboard instruments. He has also made some computer music and choral singing.

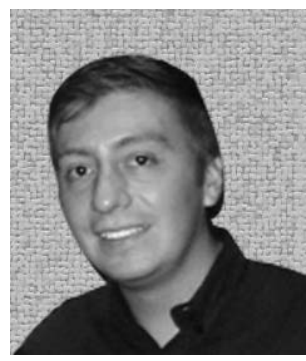

\section{Héctor Alfonso CORDOURIER-MARURI}

$\mathrm{He}$ is a PhD student of electrical engineering at the CCADET-UNAM. He has a BSc degree in engineering physics (Universidad Autónoma de Yucatan, Mexico, 2004), and an MSc degree in electrical engineering (UNAM, Mexico, 2006). He has made research in the areas of musical acoustics and sound instrumentation, and currently works in the area of active control of sound and digital signal processing.

An amateur musician and writer, he plays keyboard instruments and also has published one fiction novel. 